\title{
Determination of Crystal Structure from Electron Backscatter Diffraction Patterns
}

\author{
Stuart I. Wright, * David J. Dingley,** and Matthew M. Nowell* \\ * EDAX-TSL, 392 E 12300 S, Suite H, Draper, UT 84020 USA \\ ** Nortons Retreat, Nortons Wood Lane, Clevedon, Somerset B5217AE, UK
}

Electron Backscatter Diffraction (EBSD) is primarily used for the determination and mapping of crystallographic orientation of crystalline materials of know phase. However, it is also used to determine the phase. The general approach is to obtain the chemical composition via Energy Dispersive Spectroscopy (EDS). This information is then used as a filter against a crystallographic database. The crystal structure parameters for all of the candidate phases are then used to attempt to index the EBSD pattern. The candidate phases are then ranked according to the fit they produce with respect to the EBSD pattern. This method often works well; however, if the phase is not in the database then the approach fails. In this case, it would be helpful to be able to extract the pertinent crystallographic parameters such as the symmetry and lattice parameters $(a, b \& c$ and $\alpha, \beta \& \gamma)$ from the pattern itself. This presentation outlines a potential approach to extracting such information from an individual EBSD pattern. [1]

The basic approach is composed of three steps: (1) construct a triclinic unit cell from the pattern; (2) determine the crystal symmetry from an examination of the triclinic cell, and (3) determine the lattice parameters.

The triclinic unit cell is constructed by finding at least three bands passing through two zone axes in the pattern. One of the bands must be common to both zone axes. A three dimensional triclinic unit cell is constructed based on the identified bands as shown in figure 1. The EBSD pattern is indexed in terms of the triclinic cell thus formed. Because of the symmetry of the crystal, the pattern indexing results in independent multiple orientations related through the symmetry of the crystal. The relationship between these multiple orientations can be used to determine the symmetry of the crystal system. For example, if two of the orientations are related by a $120^{\circ}$ rotation, then the symmetry of the underlying crystal system is cubic. Once the crystal symmetry is establish the lattice parameters can be determined by comparing simulated diffraction bands with those in the EBSD pattern.

References

[1] D.J. Dingley and S.I. Wright, J. Appl. Cryst., in press. 

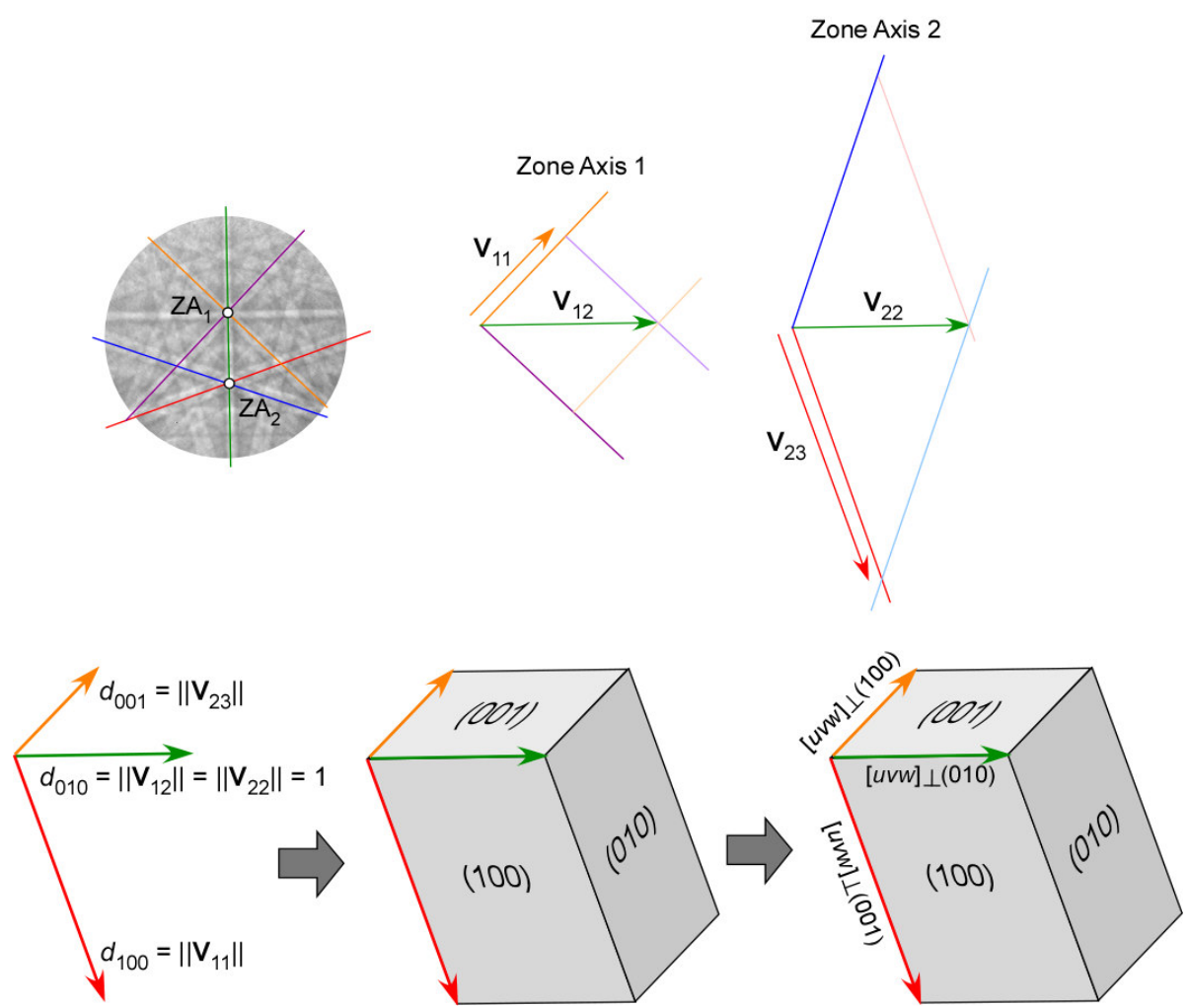

FIG. 1. Triclinic unit cell constructed from bands in a diffraction pattern.
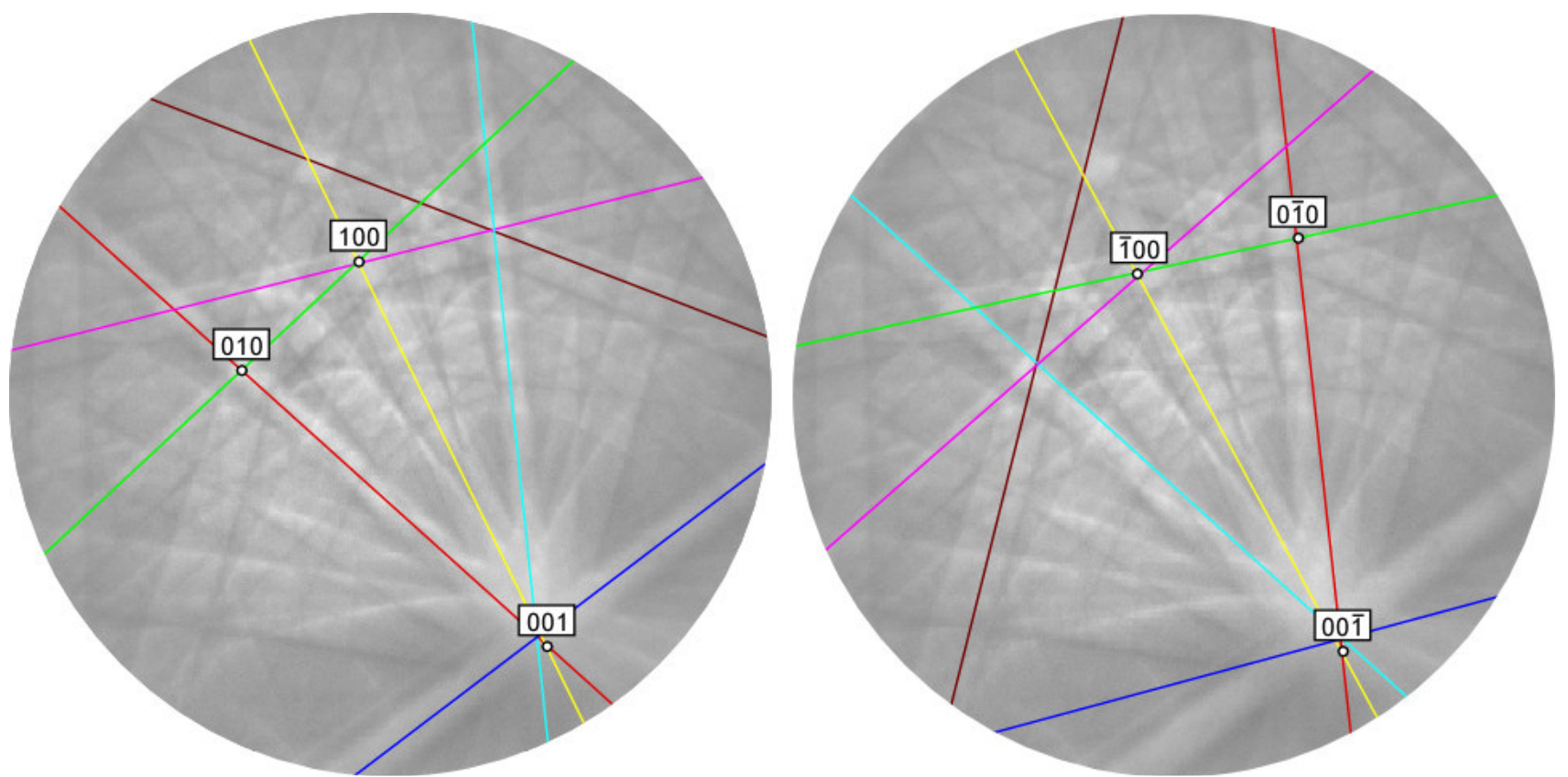

FIG. 2. Two indexing solutions for a pattern from titanium based on the derived triclinic unit cell. These two patterns are related by a $180^{\circ}$ rotation. 